\title{
A STAR-LIKE CIRCUIT-SWITCHING NETWORK WITH MULTISTAGE SERVICE
}

\author{
Z. KHALIL AND \\ G. FALIN*, Concordia University
}

\begin{abstract}
A star-like circuit-switching network with multistage service is considered. Likelihood ratio ordering is used to show that there exists an optimal threshold for the mean number of messages processed through the network as a function of the input traffic intensity.
\end{abstract}

LIKELIHOOD RATIO ORDERING; OPTIMAL NETWORK PERFORMANCE

\section{Introduction}

In the theory of circuit-switching networks with losses, only networks with one-stage service have been investigated. One-stage service means that at the moment of arrival of a call it is possible to know immediately whether or not a route exists for information transmission between calling and terminal nodes. Accordingly the call immediately occupies the route and starts to be served or leaves the system (see, for example, [1], [2]).

In the present note we consider a star-like circuit-switching network with multistage service. This means that a call successively occupies lines at a preassigned route and gets a busy signal in the case of blocking of some line only at the moment of appearance at its entry. The central mode may represent an operator connecting calls between different sources and destinations. The model also may represent multimedia services in an ATM network. In such networks we show that an interesting phenomenon is taking place: namely, the number of calls processed tends to decrease when the traffic load is too large.

\section{Model description}

Consider a network with one central node $v_{0}$ and $K$ peripheral nodes $v_{1}, \cdots, v_{K}$. Each of the peripheral nodes is connected to the central node by a single channel, so it is a network with $K$ links each with capacity 1 . Assume an input Poisson process of calls with rate $a$ at each node $v_{i}, 1 \leqq i \leqq K$; all these processes are mutually independent. A call, arriving at node $i$, at first analyses the state of the line between $v_{i}$ and $v_{0}$. If the line is engaged, the call is lost. If the line is free then the call immediately occupies it for a random period of time (first-stage service). After the service the call chooses the destination node $v_{j}$ randomly (i.e. with probability $1 /(K-1)$ as destination any node $v_{j}, j \neq i$, can be chosen) and analyzes the state of the line between $v_{0}$ and $v_{j}$. If the line is busy the call is lost and the earlier occupied line between $v_{i}$ and $v_{0}$ becomes idle. If the line is free then the call immediately occupies it for a random period of time (second-stage service), keeping the earlier occupied line between $v_{i}$ and $v_{0}$ engaged as well. At the end of the second-stage service the call leaves the network and both lines become free.

We assume that first-stage service times have the same exponential distribution with parameter $\mu_{1}$ and second-stage service times have the same exponential distribution with

Received 19 July 1990; revision received 5 September 1990.

Research partially supported by Natural Science and Engineering Research Council of Canada grant \#A9045.

${ }^{*}$ Postal address for both authors: Department of Mathematics and Statistics, Concordia University, Montreal, Quebec, Canada H4B 1R6. 
parameter $\mu_{2}$. As usual we suppose that all random variables involved are mutually independent.

Let $v_{1}(t), v_{2}(t)$ be the number of calls in progress in the first and second stages respectively. The two-dimensional process $\left(v_{1}(t), v_{2}(t)\right)$ is a Markov process with finite state-space $S=\left\{(i, j) \in Z_{t}^{2} \mid i+2 j \leqq K\right\}$ (each call at a second stage holds two lines). From a point $(i, j) \in S$ we can reach only the following points (below $\chi\left(^{\prime}\right)$ is the indicator function of a set $S$, i.e. $\chi(i, j)=1$ if $(i, j) \in S$ and $\chi(i, j)=0$ otherwise):

1. $(i+1, j)$ with rate $a(K-i-2 j) \chi(i+1, j)$;

2. $(i-1, j)$ with rate $i \mu_{1}(i+2 j-1) /(K-1)$;

3. $(i-1, j+1)$ with rate $i \mu_{1} \chi(i-1, j+1)(K-i-2 j) /(K-1)$;

4. $(i, j-1)$ with rate $j \mu_{2}$.

In what follows we consider the network in steady state (which exists due to the finiteness of the state space).

\section{Main result}

The above transition intensities of the process $\left(v_{1}(t), v_{2}(t)\right)$ allow us to write the Kolmogorov equations for steady-state probabilities $p_{i j}=\lim _{t \rightarrow \infty} P\left(v_{1}(t)=i, v_{2}(t)=j\right)$. By direct substitution in these equations one can check that the probabilities

$$
p_{i j}=\frac{a^{i+j}}{\mu_{1}^{i} \mu_{2}^{j}(K-1)^{j}} \frac{K !}{i ! j !(K-i-2 j) !} p_{\infty}, \quad 0 \leqq i+2 j \leqq K
$$

satisfy the set of equations. Here $p_{\infty}$ can be determined from the normalizing condition.

In particular, for the steady-state distribution $p_{j}=\lim _{t \rightarrow \infty} p\left(v_{2}(t)=j\right)$ of the number of calls processed at the second stage of service we have

$$
p_{j}=\left[\frac{a}{\mu_{2}(K-1)\left(1+a / \mu_{1}\right)^{2}}\right]^{2} \frac{K !}{j !(K-2 j) !} p_{0}, \quad 0 \leqq j \leqq \frac{K}{2},
$$

where $p_{0}$ can be determined from the normalizing condition.

From this we can find $Y=\lim _{t \rightarrow \infty} E v_{2}(t)=\sum_{j} j p_{j}$. This value determines the mean profit per unit time from the network functioning (because it is the only time during which information carried through is profitable).

Numerical calculations showed that the dependence of $Y$ on traffic load $a$ at each node is as shown in Figure 1.

We show below this dependence really has the same form for a network with any number of peripheral nodes. Moreover we can establish the stronger result on the increasing and decreasing nature of the distribution $p_{j}$ dependent on the parameter $a$. For this purpose we use the stochastic ordering $\leqq_{\mathrm{r}}$. Recall that if $\left(p_{j}^{\prime}\right),\left(p_{j}^{\prime \prime}\right)$ are any distinct distributions, then $\left(p_{j}\right) \varliminf_{1 \mathrm{r}}\left(p_{j}^{\prime \prime}\right)$ iff for all $j p_{j}^{\prime} / p_{j-1}^{\prime} \leqq_{j}^{\prime \prime} / p_{j-1}^{\prime \prime}$. It is well known that $\left(p_{j}^{\prime}\right) \varliminf_{1 r^{\prime}}\left(p_{j}^{\prime \prime}\right)$ implies that $\left(p_{j}^{\prime}\right) \leqq_{\mathrm{st}}\left(p_{j}^{\prime \prime}\right)$ and this in turn implies the numerical inequality for the corresponding mean values:

$$
\sum_{j} j p_{j}^{\prime} \leqq \sum_{j} j p_{j}^{\prime \prime}
$$

(For more details, see [3].) In our case,

$$
p_{j} / p_{j-1}=\frac{a}{\left(1+a / \mu_{1}\right)^{2}} \frac{(K-2 j+1)(K-2 j+2)}{\mu_{2}(K-1) j} .
$$

The function $\varphi(a)=a /\left(1+a / \mu_{2}\right)^{2}$ is increasing for $0 \leqq a \leqq \mu_{1}$ and then is decreasing when $\mu_{1} \leqq a+\infty$. This implies that the distribution $p_{j}=p_{j}(a)$ at first increases relative to the stochastic ordering $\leqq_{1 \mathrm{r}}$ (on the interval $0 \leqq a \leqq \mu_{1}$ ) and then decreases relative to the stochastic ordering $\leqq_{1 \mathrm{r}}$ (on the interval $\left.\mu_{1} \leqq a<+\infty\right)$. From this, the corresponding behaviour of $Y(a)$ easily follows. 


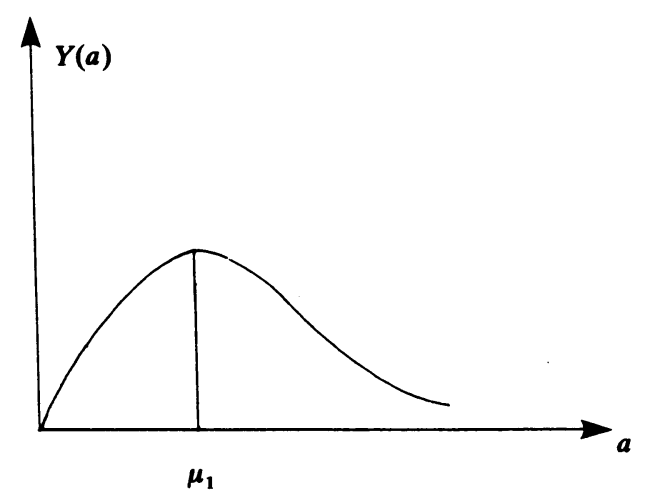

Figure 1

\section{Applications}

The above result about the existence of a maximum for $Y(a)$ seems to be of interest for optimal control of the network. If the objective is to increase the profit from the network functioning one must find ways to increase the flow of calls at the peripheral nodes. However, this can be done only up to a certain threshold (maximum of $Y(a)$ ). Further increase in input flow intensity (and thus resulting decrease in $Y(a)$ ) will be detrimental to the network. Calls will tend to compete for lines to transmit through; hence there is a decrease in the actual number of transmitted messages, and this in turn influences the profit. In our view a similar situation can also occur in other structures of multistage networks. However, the determination of the optimal value $a$ of the input intensity could be difficult. We mention specifically the fact that the phenomenon described in the above investigation cannot be observed in the models of circuit-switching networks with one-stage service.

\section{References}

[1] Burman, D. Y., LehoczKy, J. P. AND Lim, Y. (1984) Insensitivity of blocking probabilities in a circuit-switching network. J. Appl. Prob. 21, 850-859.

[2] Hunt, P. ANd Kelly, F. P. (1989) On critically loaded loss networks. Adv. Appl. Prob. 21, 831-841.

[3] Ross, S. M. (1983) Stochastic Processes. Wiley, New York. 